\title{
Multi-detector computer tomography venography (MDCTV) as a diagnostic tool in the management of patients with atypical, complicated and/or recurrent varicose veins
}

\section{A Lawson, P Rischbieter, J Owen, T Peedikayil, S Beningfield}

Department of Radiology, Groote Schuur Hospital, Cape Town

A Lawson, MB BCh, FC Rad Diag (SA), MMed Rad

P Rischbieter, MB BCh

J Owen, MB BCh

T Peedikayil, MB BCh

S Beningfield, MB ChB, FFRad (D) SA

Corresponding author: A Lawson (drajlawson@gmail.com)

\begin{abstract}
Aim. To evaluate the role of multi-detector computer tomography venography (MDCTV), compared with conventional venography, as a diagnostic tool in the management of patients with atypical, complicated and/or recurrent varicose veins.

Materials and methods. Retrospective review of 21 patients who had undergone both MDCTV and conventional transfemoral or transpopliteal venography between January 2008 and April 2011 for the management of recurrent varicose veins and/or chronic venous ulcers. MDCTV was performed using a 16-slice CT scanner. Spiral acquisition was commenced 180 seconds after intravenous injection of $150 \mathrm{ml}$ of $350 \mathrm{mmol} / \mathrm{l}$ iodinated contrast medium. A reconstruction interval of $1.5 \mathrm{~mm}$ was used. Conventional venography was performed by the resident vascular surgeon and was followed by stenting or coiling where appropriate.

Results. MDCTV and venography were compared in 21 patients (6 male, 15 female; average age 55 years, range 33 - 78 years); 8 also underwent endovascular iliac vein stenting. The area under the receiver operator curve (ROC) for percentage iliac vein stenosis determined on MDCTV versus venography was 0.75 . Four (19\%) false-positive iliac vein stenoses were reported on MDCTV. Ten patients underwent gonadal vein coil embolisation. Gonadal vein size $>5.2 \mathrm{~mm}$ (range $1-11 \mathrm{~mm}$ ) on MDCTV predicted significant venographic reflux requiring coil embolisation. Three (30\%) patients who underwent embolisation did not have gonadal vein enlargement on MDCTV.

Conclusion. MDCTV plays an important adjunctive role in the diagnostic workup of patients with complex venous disease. The findings at MDCTV correlate well with conventional venography.
\end{abstract}

S Afr J Rad 2012;16(4):136-138. DOI:10.7196/SAJR.756

Varicose veins is one of the most common pathologies among people around the world; since the introduction of less-invasive treatments such as laser ablation and radiofrequency thermal ablation, interest in this condition has increased. ${ }^{1}$ Routine surgical intervention remains the principal definitive management for large, unsightly varicose veins. A Trendelenburg strip is performed, entailing ligation of the common femoral vein at the saphenofemoral junction and removal of the native vein in its entirety from the leg.

A sub-group of patients develop recurrent varicose veins post surgery, or have atypical or complicated varicose veins. It is this group of patients who might benefit from multi-modality imaging to address the underlying causative lesion not addressed at the primary intervention or presentation.

We evaluated the role of multi-detector computer tomography venography (MDCTV) as a diagnostic tool in the management of this sub-group of patients, and compared it with conventional venography, which is considered the gold standard of venous imaging.

\section{Methods and materials}

We performed a retrospective review of 21 patients who had undergone both MDCTV and conventional transfemoral, transjugular or transpopliteal venography between January 2008 and April 2011 for the management of recurrent varicose veins and/or chronic venous ulcers. The study was approved by our institutional review board.

MDCTV was performed using a 16-slice CT scanner. Spiral acquisition was commenced 180 seconds after intravenous injection of $150 \mathrm{ml}$ of $350 \mathrm{mmol} / \mathrm{l}$ iodinated contrast medium. A reconstruction interval of $1.5 \mathrm{~mm}$ was used. Subset data reconstruction was performed in the curved coronal plane with particular reference to the course of the common and external iliac veins through the pelvis. Axial venous 
calibre measurements were performed and stenotic segments were measured on the reconstructed images.

Readers were blinded to the findings from the conventional venography datasets. Secondary imaging outcomes were to identify anatomical anomalies, pelvic masses or compression of venous structures by native vessels. Conventional venography was performed by the resident vascular surgeon. After a diagnostic flush, endovascular stenting (Figs 1 and 2) or coiling was performed if a significant stenosis was verified or significant venous reflux was demonstrated.

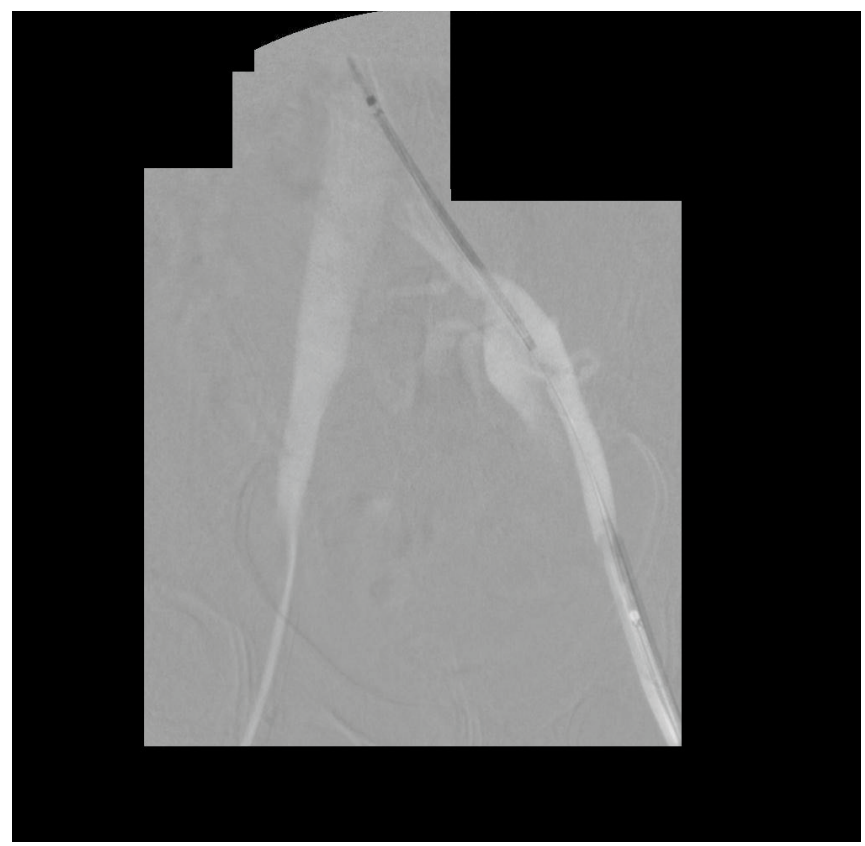

Fig. 1. Selected frame of a subtracted venogram with self-expanding metallic stent across the left common iliac vein stenosis pre-deployment.

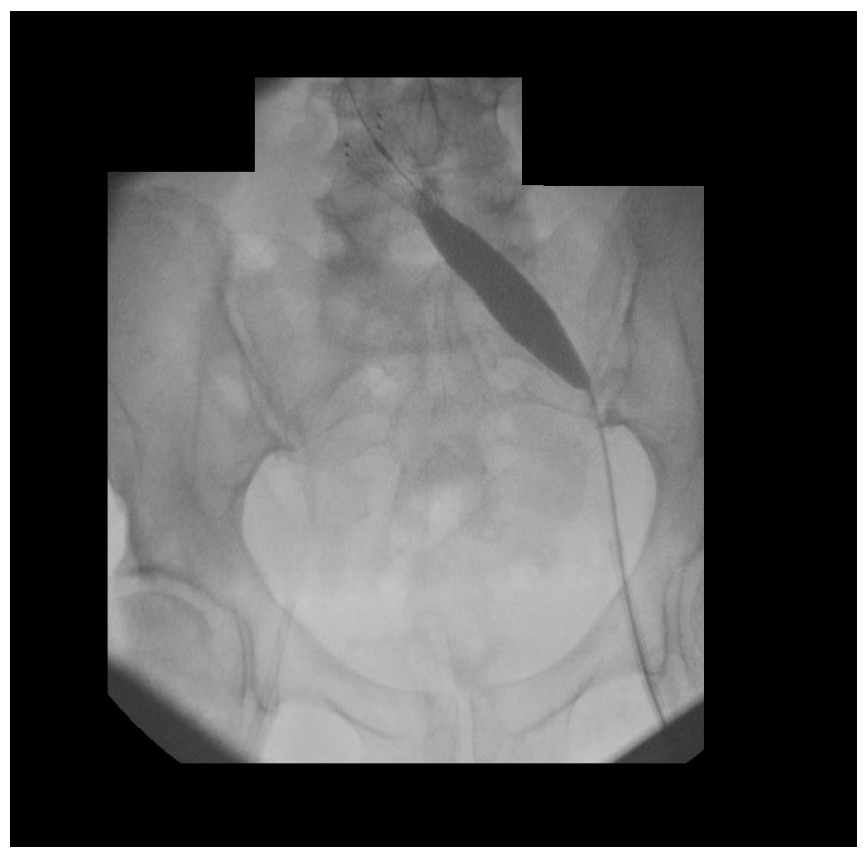

Fig. 2. Frame grab during deployment and balloon dilatation of the stent in Fig. 1.

\section{Results}

MDCTV and venography were compared in 21 patients (6 male, 15 female; average age 55 years, range 33 - 78 years); 8 also underwent endovascular iliac vein stenting. The area under the receiver operator curve (ROC) for percentage iliac vein stenosis determined on MDCTV versus venography was 0.75 . Four false-positive (19\%) iliac vein stenoses were reported on MDCTV. Aetiologically, these stenoses were attributed to post-phlebitic changes in 6 patients, May-Thurner syndrome in 1 patient, and were idiopathic in 1 patient (Fig. 3). Ten patients underwent gonadal vein coil embolisation. Gonadal vein size $>5.2 \mathrm{~mm}$ (range 1 - 11 $\mathrm{mm}$ ) on MDCTV predicted significant venographic reflux requiring coil embolisation. Three (30\%) patients who underwent embolisation did not have gonadal vein enlargement on MDCTV.

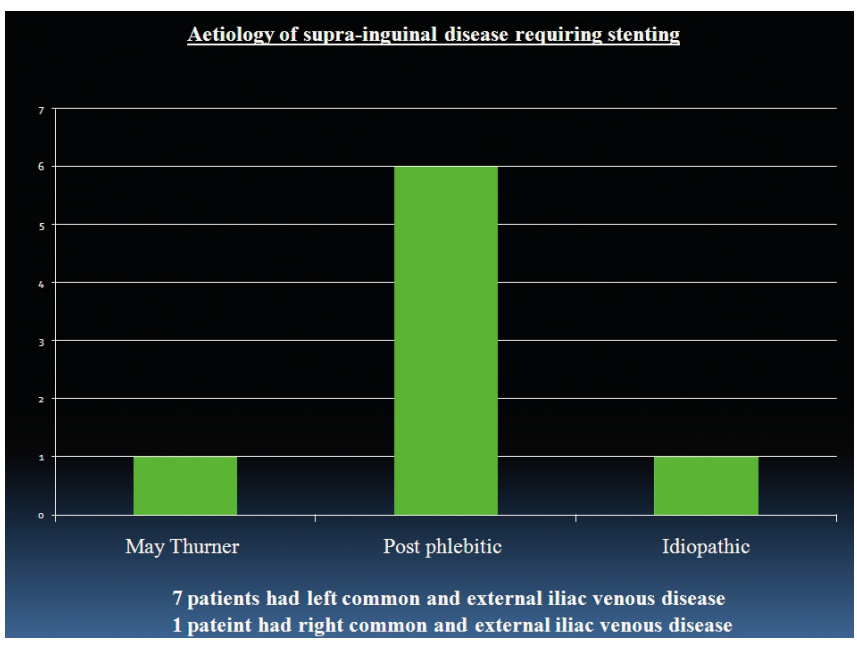

Fig. 3. Aetiology of iliac vein stenosis.

\section{Conclusion}

Currently, limited data exist on the use of MDCTV and its role in complex varicose venous disease. We present a small cohort of cases where MDCTV findings correlate well with conventional venographic findings. Our study focuses on a subset of patients with complex venous disease where the underlying causative lesions are above the level of the inguinal canal and involve mainly the common/external iliac veins or gonadal veins. The mainstay of imaging was to reveal pathology that could be managed endovascularly either by iliac vein stenting ${ }^{2,3}$ or gonadal vein coil embolisation. Preoperative evaluation of varicose veins must reveal the primary cause of varicosity, secondary reflux, and the distribution of varicosity, including perforating veins. ${ }^{1}$

Handheld Doppler and duplex sonography have become the modern non-invasive gold standard. ${ }^{4}$ Sonography can evaluate haemodynamic information and anatomical data; its main limitation is the lack of adequate visualisation above the inguinal ligament.

MDCTV provides excellent information on the deep venous system above and below the inguinal canal. Multiplanar reformations and volume rendering provide both an assessment of venous compression and overall varicosity distribution. ${ }^{5}$ The major limitation of MDCTV is the lack of dynamic venous flow information, limiting the interrogation of points of reflux. Furthermore, MDCTV requires contrast medium administration and ionising radiation $\left(1.6-3.9 \mathrm{mSv}^{1}\right)$ and no insight to venous valvular function can be gained. 


\section{CASE SERIES}

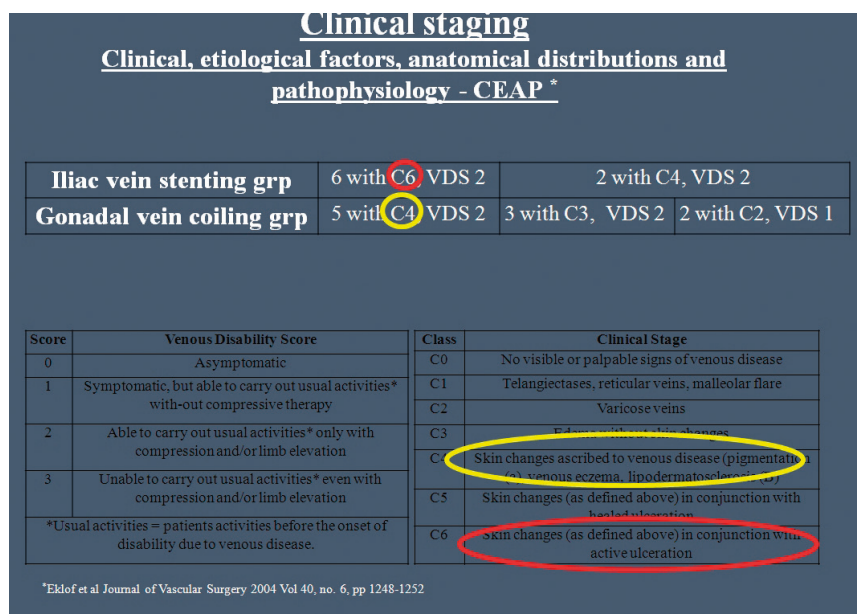

Fig. 4. Clinical disease severity index.

A clinical scoring system documenting the severity of venous disease is described by Eklöf et al. ${ }^{6}$ (Fig. 4). In our study, patients with clinical stage C6 venous disease were more likely to require iliac stenting. It was in this group that an almost $20 \%$ false-positive rate was recorded at MDCTV. In light of the clinical severity score, these patients may still benefit from diagnostic conventional venography with the adjunctive use of endovascular ultrasound to delineate potential stenoses missed at both conventional catheter venography and MDCTV. Patients with C4 disease were more likely to require gonadal vein coiling. Selecting the latter group of patients purely on MDCTV findings is not recommended.

Varicose venous disease is a complex entity; careful patient selection and intervention is required for successful and curative management. This study indicates that, although MDCTV contributes to the multi-modality approach in venous disease, more extensive work needs to be done before it can play a larger role in definitive interventional decision making.

1. Lee W, Chung JW, Yin YH, et al. Three-dimensional CT venography of varicose veins of the lower extremity: Image quality and comparison with Doppler sonography. Am J Roentgenol 2008;191(4):11861191.

2. Titus JM, Moise MA, Bena J, Lyden SP, Clair DG. Iliofemoral stenting for venous occlusive disease. J Vasc Surg 2011;53(3):706-712.

3. Rosales A, Sandbæk G, Jørgensen IJ. Stenting for chronic post-thrombotic vena cava and iliofemora venous occlusions: Mid-term patency and clinical outcome. Eur J Vasc Endovasc Surg 2009;40(2):234240.

4eissner MH, Eklof B, Smith PC, et al. Secondary chronic venous disorders. J Vasc Surg 2007;46(6, Supplement 1):S68-S83.

5. Min S-K, Kim S-Y, Park YJ, et al. Role of three-dimensional computed tomography venography as a powerful navigator for varicose vein surgery. J Vasc Surg 2010;51(4):893-899.

6. Eklöf B, Rutherford RB, Bergan JJ, et al. Revision of the CEAP classification for chronic venous disorders: Consensus statement. J Vasc Surg 2004;40(6):1248-1252.

\section{Correction notice}

We regret that an error occurred in the diagnostic algorithm on page 119 of the September 2012 issue (Vol. 16 No. 3) of the SAJR. The phrases 'No air-trapping on exp. HRCT' and 'Air-trapping on exp. HRCT' in the 2nd and 3rd columns of the centre row were inadvertently transposed. The corrected image has been placed in the online version of the journal (URL http://www.sajr.org.za/index.php/sajr/article/view/769/621). We apologise for this error. 